\title{
Effects of Polyvinyl Alcohol on Drug Release from Nanocomposite Particles Using Poly (L-lactide-co-glycolide)
}

\author{
Issei Takeuchi ${ }^{1,2 *}$, Yuuto Kato ${ }^{1}$, and Kimiko Makino,2* \\ ${ }^{1}$ Faculty of Pharmaceutical Sciences, Tokyo University of Science, 2641, Yamazaki, Noda, Chiba 278-8510, JAPAN \\ ${ }^{2}$ Center for Drug Delivery Research, Tokyo University of Science, 2641, Yamazaki, Noda, Chiba 278-8510, JAPAN
}

\begin{abstract}
The effects of polyvinyl alcohol (PVA) on the release behavior of polymer nanoparticles from nanocomposite particles using amino acids were investigated. Rifaximin (RFX) was used as a hydrophobic drug model. RFX-loaded poly(L-lactide-co-glycolide) (PLLGA) nanoparticles were prepared using an antisolvent diffusion method. They were then spray-dried with equal amounts of amino acids to prepare the nanocomposite particles. The mean diameters of nanocomposite particles were $2.86-5.42 \mu \mathrm{m}$. The particle size increased as the concentration of PVA aqueous solution increased. The mean diameters of RFX-loaded PLLGA nanoparticles were $150-160 \mathrm{~nm}$; however, the particle size distributions of those prepared using $0.25 \%(w / v)$ PVA aqueous solution differed significantly immediately after preparation and after redispersion from nanocomposite particles. The release test results of nanocomposite particles revealed that those prepared using $0.25 \%$ and $0.50 \%(w / v)$ aqueous PVA solutions rapidly released RFX. In contrast, particles prepared using 2.00 and $4.00 \%$ (w/v) PVA aqueous solution showed sustained drug release. The results of drug release tests of nanoparticles redispersed from nanocomposite particles showed that the nanoparticles prepared using $0.50 \%$ and $2.00 \%$ (w/v) PVA aqueous solution suppressed the initial burst. Therefore, we considered that the results of the drug release behavior of the nanoparticles in these particles reflectsreflect the release behavior of the nanoparticles from the nanocomposite particles. These results indicate that the rate of redispersion from nanocomposite particles to nanoparticles can be controlled by changing the concentration of PVA aqueous solution.
\end{abstract}

Key words: poly(L-lactide-co-glycolide), nanoparticles, nanocomposite particles, polyvinyl alcohol, amino acids

\section{Introduction}

Parenteral drug administration has attracted attention in drug delivery systems. Bioavailability can be improved by avoiding the hepatic first-pass effect, which can prematurely metabolize drugs ${ }^{1,2)}$. Inhalation systems are one of the non-invasive and self-administering methods. Inhalation formulations are a promising system for systemic administration because of the large surface area of the lungs and thin absorption barrier ${ }^{3)}$. In topical administration for the treatment of pulmonary diseases, they can deliver drugs directly and rapidly to the diseased lung tissue site, which is advantageous over oral formulations in which only a small proportion is delivered to the diseased site ${ }^{4)}$. To efficiently deliver the drug to the target site, it is necessary to control the size and shape of the inhalation formulation. It has been reported that particles with a mean aerody- namic diameter of 1-3 $\mu \mathrm{m}$ are deposited minimally in the mouth and throat and maximally in the lung parenchymal regions, such as the alveolar or deep-lung region ${ }^{5}$. Thus, inhalable nanocomposite particles are a promising inhalation system. Furthermore, nanoparticles can overcome mucus clearance ${ }^{6)}$. In addition to macrophages, they are also taken up by other cells such as cancer cells and epithelial cells ${ }^{7-10)}$. These properties of nanoparticles are useful for drug delivery; however, the size of nanoparticles is not suitable for inhalation. Nanocomposite particles with an aerodynamic diameter suitable for inhalation are used to solve this problem. Since these particles use diluents soluble in the alveoli lining fluid, they are decomposed into nanoparticles after reaching the alveoli ${ }^{10)}$. Recently, it was reported that nanocomposite particles for inhalation improved the concentration of drug in the lungs. These

\footnotetext{
*Correspondence to: Issei Takeuchi, Faculty of Pharmaceutical Sciences, Tokyo University of Science, 2641, Yamazaki, Noda, Chiba 278-8510, JAPAN. Kimiko Makino, ibid.

E-mail: takeuchi@rs.tus.ac.jp (IT); makino@rs.tus.ac.jp (KM)

Accepted November 25, 2020 (received for review October 26, 2020)

Journal of Oleo Science ISSN 1345-8957 print / ISSN 1347-3352 online

http://www.jstage.jst.go.jp/browse/jos/ http://mc.manusriptcentral.com/jjocs
} 
studies with nanocomposite particles containing sildenafil $^{11)}$ and rifampicin ${ }^{12)}$-loaded nanoparticles demonstrate the usefulness of transpulmonary administration using polymer nanoparticles. Polymer nanoparticles are useful carriers because of their biocompatibility, biodegradability, ease of surface modification, localization, and reduced systemic toxicity ${ }^{13)}$. In addition, they are capable of controlling drug release behavior. In previous studies, we investigated the physicochemical properties that affect the drug release behavior of nanoparticles prepared using poly (lactide-coglycolide ${ }^{14,15)}$. In studies using curcumin ${ }^{4}$, tadalafil $^{16)}$, and simvastatin $^{17)}$, nanocomposite particles containing drug nanoparticles were prepared using the spray drying method. It was reported that nanocomposite particle formation increased solubility, mainly due to the amorphization of the drug. Studies have been conducted on the behavior of drug release from nanocomposite particles, whereas there are few studies on the behavior of the release of nanoparticles from them. While sugars have conventionally been used as diluents for nanocomposite particles, amino acids have recently attracted attention for improving pulmonary delivery in high humidity ${ }^{18-20)}$. In general, amino acids have a lower solubility in water than sugars. Hence, research focusing on their dissolution behavior is necessary.
In this study, we controlled the release behavior of poly (L-lactide-co-glycolide) (PLLGA) nanoparticles from nanocomposite particles using polyvinyl alcohol(PVA). PVA is used in various pharmaceutical applications, such as stabilizing agents for emulsions ${ }^{21)}$, artificial tears ${ }^{22)}$, and sustained-release formulations for oral administration ${ }^{23)}$. An evaluation of oral toxicity showed that this additive was safe even at high levels of consumption ${ }^{24)}$. The nanoparticle surfaces are easily coated with PVA using an aqueous PVA solution $^{25)}$. We considered that the PVA-derived hydrated layer on the nanoparticles' surface caused an efficient influx of water into the nanocomposite particles and aided in the redispersion of the nanoparticles. PLLGA was suitable for this study because it can prepare nanoparticles with slower drug release than those using PLGA ${ }^{14,15)}$. Rifaximin (RFX), a hydrophobic antibacterial agent, was used as a model $\mathrm{drug}^{26)}$. Release tests were performed on the nanocomposite particles to investigate nanoparticles' release behavior after reaching the lungs. Similarly, release tests were performed on nanoparticles redispersed from nanocomposite particles to investigate drug release behavior after reaching the lungs. The relationship between nanoparticles, nanocomposite particles, and nanoparticles redispersed from the nanocomposite particles is shown in Fig. 1.
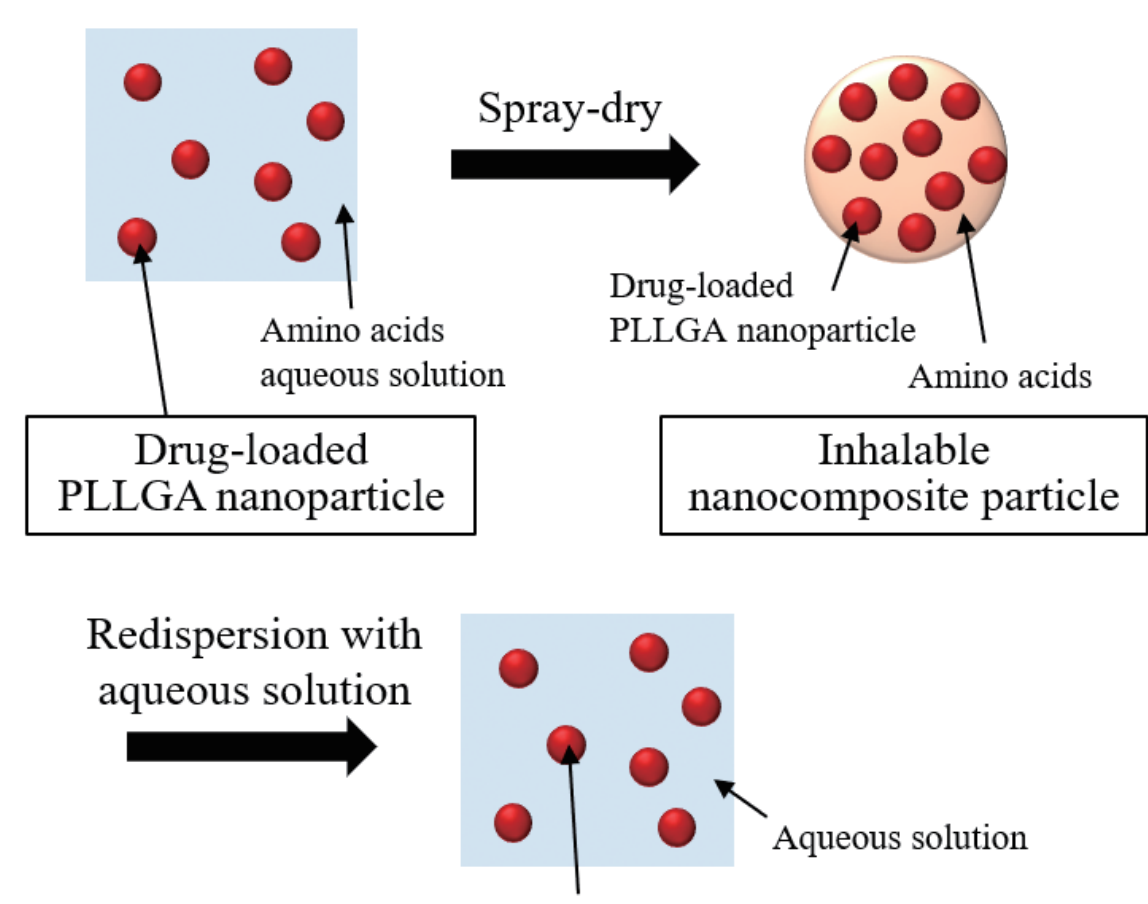

Drug-loaded PLLGA nanoparticle

Nanoparticles redispersed
from nanocomposite particles

Fig. 1 Schematic diagram of the relationship between nanoparticles, nanocomposite particles, and nanoparticles redispersed from nanocomposite particles. 


\section{Effects of Polyvinyl Alcohol on Nanocomposite Particles}

\section{Experimental Procedures}

\subsection{Materials}

PLLGA with a molecular weight of 10,000 and an L-lactic acid/glycolic acid monomer composition of 75/25 was purchased from Taki Chemical Co., Ltd. (Kakogawa, Japan). $\operatorname{RFX}\left(\mathrm{C}_{43} \mathrm{H}_{51} \mathrm{~N}_{3} \mathrm{O}_{11}\right.$, purity $\left.\geq 98.0 \%\right)$, polyvinyl alcohol(PVA, degree of polymerization: 500), ethanol (EtOH, purity $\geq$ $99.5 \%)$, acetone $\left(\mathrm{CH}_{3} \mathrm{COCH}_{3}\right.$, purity $\left.\geq 99.5 \%\right), \mathrm{L}(+)$-arginine hydrochloride (purity $\geq 99.0 \%$ ), and L-leucine (purity $\geq 99.0 \%$ ) were purchased from Fujifilm Wako Pure Chemical Corp. (Osaka, Japan). Acetonitrile $\left(\mathrm{CH}_{3} \mathrm{CN}\right.$, for HPLC, purity > 99.9\%) was purchased from Kanto Chemical Co., Inc. (Tokyo, Japan). All other chemicals were of the highest grade commercially available.

\subsection{Preparation of RFX-loaded PLLGA nanoparticles}

RFX-loaded PLLGA nanoparticles were prepared using an antisolvent diffusion method ${ }^{25,27)}$. Briefly, $190 \mathrm{mg}$ of PLLGA and $10 \mathrm{mg}$ of RFX were dissolved in $4 \mathrm{~mL}$ of a mixed solution of acetone and ethanol (acetone:ethanol $=$ $5: 3)$. The solution was injected into $40 \mathrm{~mL}$ of purified water, 0.25, 0.50, 2.00, and 4.00\% (w/v) PVA aqueous solution, and then the RFX-loaded PLLGA nanoparticles were immediately precipitated. The suspension was dialyzed for $24 \mathrm{~h}$ in a dialysis tube (C36-32-100, molecular weight cutoff: 14,000, EIDIA Co., Ltd., Tokyo, Japan) to remove unloaded-RFX. Purified water was used as the external liquid and was replaced after 2, 4, 6, and $8 \mathrm{~h}$. After dialysis, RFXloaded PLLGA nanoparticle suspensions were obtained. The mean volume diameters and size distributions of the nanoparticles were determined using a dynamic light scattering system(ELSZ-2, Otsuka Electronics Co., Ltd., Hirakata, Japan), which measures the scattered light that is generated when the laser light is irradiated onto particles that are in Brownian motion at $25^{\circ} \mathrm{C}^{28)}$.

\subsection{Preparation of nanocomposite particles}

The preparation of nanocomposite particles was performed similar to our previous study ${ }^{19)}$. The nanoparticles were redispersed in purified water, and a physical mixture of arginine hydrochloride and leucine (arginine hydrochloride:leucine $=1: 6$ ) was added to an equal weight of precipitated nanoparticles. The suspensions were then spraydried to prepare nanocomposite particles using a spray dryer (Mini Spray Dryer B-290, BÜCHI Corp., Flawil, Switzerland). Spray drying was performed under the following conditions: an outlet temperature of $37-40^{\circ} \mathrm{C}$, an air volume of $22.5 \mathrm{~m}^{3} / \mathrm{h}$, and a pump flow rate of $1.4 \mathrm{~mL} / \mathrm{min}$. After freezing at $-30^{\circ} \mathrm{C}$, the nanocomposite particles were lyophilized using a freeze dryer (FD-1000, Tokyo Rikakikai Co., Ltd., Tokyo, Japan) for 12 h. The size of the nanocomposite particles in the air was measured using a sizer (LDSA-3500A, Nikkiso Co., Ltd., Tokyo, Japan). The mean volume diameters and the size distributions of PLLGA nanoparticles redispersed from the nanocomposite particles were determined using a dynamic light scattering system(ELSZ-2) at $25^{\circ} \mathrm{C}$. RFX content in the particles was measured using high-performance liquid chromatography (HPLC, SIL-20A prominence, SPD-20A prominence, LC-20AD prominence, CTO-10ASvp, DGU-20A3 prominence, Shimadzu Co., Kyoto, Japan) at $290 \mathrm{~nm}$ with an ODS column (STR ODS-M, size: $4.6 \mathrm{~mm} \times 150 \mathrm{~mm}$, Shinwa Chemical Industries Ltd., Kyoto, Japan). The mobile phase consisted of $52 \%$ water, $0.1 \%$ acetic acid, and $48 \%$ ethanol. Samples were filtered through a $0.45 \mu \mathrm{m}$ pore membrane (Tokyo Roshi Kaisha, Ltd., Tokyo, Japan). The samples were dissolved in $10 \mathrm{~mL}$ of solution. HPLC measurements were performed at ambient temperature at a flow rate of $0.9 \mathrm{~mL} / \mathrm{min}$, and $20 \mu \mathrm{L}$ of sample solution was applied. All HPLC measurements were performed under the same conditions ${ }^{26)}$.

\subsection{In vitro release study of RFX from nanocomposite particles}

To evaluate the effect of PVA on nanocomposite particles' drug release behavior, the release ratios of RFX from nanocomposite particles were determined. The cumulative release ratio was expressed as the ratio of RFX released from the particles divided by the amount of RFX initially contained in the nanocomposite particles ${ }^{29)}$. The particles were added to $500 \mathrm{~mL}$ of phosphate-buffered saline(PBS, $\mathrm{pH}$ 7.4) in a vessel to an RFX concentration of $1.0 \mu \mathrm{g} / \mathrm{mL}$. Release tests were performed using a dissolution tester (NTR-6100A, Toyama Sangyo Co., Ltd., Osaka, Japan) at $100 \mathrm{rpm}$ at $37^{\circ} \mathrm{C}$. After 1, 2, 4, 6, 8, 10, 20, 30, 40, 50, and $60 \mathrm{~min}$ from the test initiation, $1.0 \mathrm{~mL}$ of each sample was collected. The cumulative release ratios of RFX from nanocomposite particles at each time point were calculated by measuring the amount of RFX using HPLC.

\subsection{In vitro release study of RFX from redispersed RFX- loaded PLLGA nanoparticles}

To evaluate the effect of PVA on drug release behavior, the release ratios of RFX from redispersed RFX-loaded PLLGA nanoparticles were determined. The cumulative release ratio was expressed as the ratio of $\mathrm{RFX}$ released from nanoparticles divided by the amount of RFX initially contained in the nanoparticles ${ }^{29)}$. Ten milligrams of nanocomposite particles were redispersed in $50 \mathrm{~mL}$ of PBS ( $\mathrm{pH}$ 7.4) using a vortex mixer (Vortex-Genie 2, Electro Scientific Industries, Inc., Portland, OR). The suspension was diluted with PBS to an RFX concentration of $0.5 \mu \mathrm{g} / \mathrm{mL}$. In total, 3 $\mathrm{mL}$ of the suspension was placed in a dialysis tube ${ }^{30)}$ and added to $97.0 \mathrm{~mL}$ of PBS (pH 7.4). The sample suspensions were shaken at $100 \mathrm{rpm}$ and incubated at $37^{\circ} \mathrm{C}$ for $0.5,1,2$, $4,8,12$, or $24 \mathrm{~h}$. The amount of RFX remaining in the dialysis membrane was quantified using HPLC. The amount of RFX released from the nanoparticles at each time was cal- 


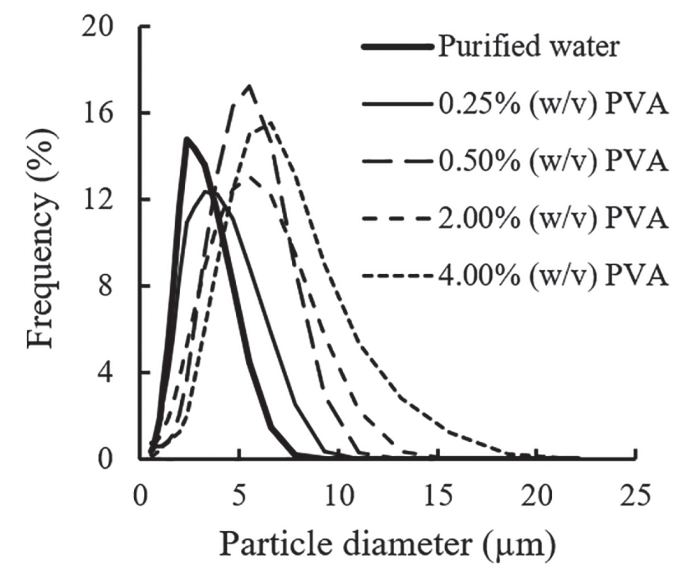

Fig. 2 Particle size distribution of nanocomposite particles containing RFX-loaded PLLGA nanoparticles $(\mathrm{n}=3)$.

culated by subtracting the measured residual amount from the initial amount ${ }^{29)}$.

\section{Results and Discussion}

\subsection{Characterization of nanocomposite particles and} RFX-loaded PLLGA nanoparticles

The mean diameters of nanocomposite particles prepared using purified water, $0.25,0.50,2.00$, or $4.00 \%(\mathrm{w} / \mathrm{v})$ PVA aqueous solution were $2.86 \pm 0.18,2.89 \pm 0.10,4.33 \pm$ $0.27,4.86 \pm 0.27$, and $5.42 \pm 1.12 \mu \mathrm{m}$, respectively. Figure 2 shows the particle size distributions of the nanocomposite particles. The particle size increased as the concentration of PVA aqueous solution increased. The aerodynamic diameter, $d_{a e r}$, of the particles is calculated using the following equation:

$$
d_{\text {aer }}=d_{\text {mass }} \sqrt{\frac{\rho}{F}}
$$

where $d_{\text {mass }}, \rho$, and $F$ are the geometrical particle diameter, density of the particle, and shape function, respectively. The spherical particles are calculated with $F=1^{31,32)}$. Therefore, an increase in the $d_{\text {mass }}$ of nanocomposite particles due to an increase in the concentration of PVA aqueous solution may reduce their intrapulmonary delivery. RFX content in the particles prepared using purified water, $0.25,0.50,2.00$, or $4.00 \%(\mathrm{w} / \mathrm{v})$ PVA aqueous solution were $2.47 \pm 0.02,2.44 \pm 0.02,2.47 \pm 0.01,2.45 \pm 0.01$, and $2.46 \pm$ $0.03 \%$, respectively. These results suggest that the concentration of PVA aqueous solution does not affect the RFX content of the particles. Figure 3 shows the particle size distributions of RFX-loaded PLLGA nanoparticles. Their mean diameters and coefficients of variation are shown in Table 1. In the nanoparticles prepared using $0.25 \%(\mathrm{w} / \mathrm{v})$ PVA aqueous solution, the redispersed nanoparticles had a smaller mean diameter and a higher
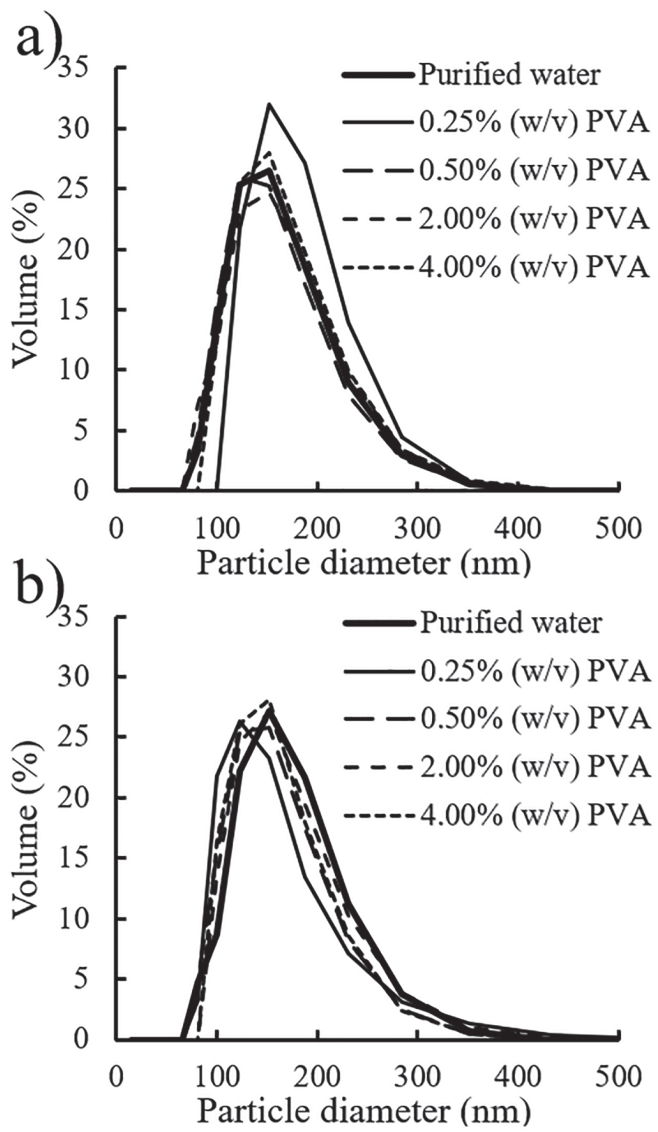

Fig. 3 Particle size distribution of RFX-loaded PLLGA nanoparticles $(n=3)$. (a) immediately after preparation. (b)after redispersion from nanocomposite particles.

coefficient of variation than the nanoparticles immediately after preparation. PLGA nanoparticles have negative charges at neutral $\mathrm{pH}$ because of the ionization of the terminal carboxyl groups of PLGA ${ }^{33)}$. This also applies to PLLGA nanoparticles, which contribute to improved dispersion stability of the particles in the solvent. In PLGA nanoparticles using PVA, it was reported that the negative charge derived from PLGA was shielded by the PVA covering the surface of the nanoparticles ${ }^{25}$. Therefore, we considered that the PVA-derived hydrated layer adsorbed on the surface contributes to the nanoparticles' dispersion stability. The instability of the nanoparticles prepared using $0.25 \%(\mathrm{w} / \mathrm{v})$ PVA aqueous solution suggests that both the negative charge and the hydrated layer on their surfaces were insufficient. In the other nanoparticles, no significant changes were observed between those immediately after preparation and those after redispersion from nanocomposite particles. From these findings, we confirmed that various nanocomposite particles and PLLGA nanoparticles were successfully prepared. 
Table 1 Mean volume diameters and coefficient of variation of PLLGA nanoparticles (mean \pm S.D., $\mathrm{n}=3$ ).

\begin{tabular}{ccccc}
\hline \multirow{2}{*}{ Poor solvent } & \multicolumn{2}{c}{$\begin{array}{c}\text { Nanoparticles immediately after } \\
\text { preparation }\end{array}$} & \multicolumn{2}{c}{$\begin{array}{c}\text { Nanoparticles after redispersion from } \\
\text { nanocomposite particles }\end{array}$} \\
\cline { 2 - 5 } & $\begin{array}{c}\text { Mean volume } \\
\text { diameter }(\mathrm{nm})\end{array}$ & $\begin{array}{c}\text { Coefficient of } \\
\text { variation }\end{array}$ & $\begin{array}{c}\text { Mean volume } \\
\text { diameter }(\mathrm{nm})\end{array}$ & $\begin{array}{c}\text { Coefficient of } \\
\text { variation }\end{array}$ \\
\hline Purified water & $160.5 \pm 49.7$ & 0.31 & $154.0 \pm 48.3$ & 0.31 \\
$0.25 \%(\mathrm{w} / \mathrm{v})$ PVA & $173.8 \pm 44.7$ & 0.26 & $149.5 \pm 55.8$ & 0.37 \\
$0.50 \%(\mathrm{w} / \mathrm{v})$ PVA & $150.4 \pm 48.5$ & 0.32 & $150.3 \pm 47.1$ & 0.31 \\
$2.00 \%(\mathrm{w} / \mathrm{v})$ PVA & $154.4 \pm 51.4$ & 0.33 & $160.6 \pm 51.1$ & 0.32 \\
$4.00 \%(\mathrm{w} / \mathrm{v})$ PVA & $159.5 \pm 48.7$ & 0.31 & $153.3 \pm 45.0$ & 0.29 \\
\hline
\end{tabular}

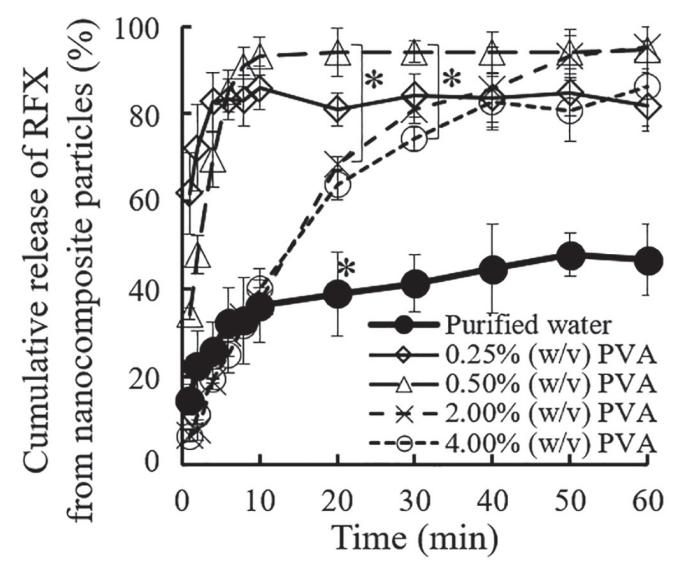

Fig. 4 Cumulative release ratios of RFX from nanocomposite particles prepared using purified water, $0.25,0.50,2.00$, and $4.00 \%(\mathrm{w} / \mathrm{v}) \mathrm{PVA}$ aqueous solution (mean \pm S.D., $\mathrm{n}=3,{ }^{*} p<0.05$, Tukey's test).

\subsection{In vitro release of RFX from nanocomposite particles}

Figure 4 shows the cumulative release ratios of RFX from nanocomposite particles prepared using purified water, $0.25,0.50,2.00$, and $4.00 \%(\mathrm{w} / \mathrm{v})$ PVA aqueous solution at $37^{\circ} \mathrm{C}$. The release ratios of the particles prepared using purified water, $0.25,0.50,2.00$, and $4.00 \%(\mathrm{w} / \mathrm{v}) \mathrm{PVA}$ aqueous solution after $10 \mathrm{~min}$ from the initiation of the tests were $36.2 \pm 8.4 \%, 85.9 \pm 4.9 \%, 93.1 \pm 4.3,37.1 \pm 3.1$, and $40.0 \pm 1.9 \%$, respectively. In the particles prepared using $0.25 \%$ and $0.50 \%(\mathrm{w} / \mathrm{v})$ PVA aqueous solution, the cumulative release ratios of RFX increased rapidly. This result suggests that a large amount of RFX was released from the nanoparticles, or the RFX-loaded nanoparticles were dispersed in the solvent due to efficient amino acid dissolution. In addition, a visual observation confirmed that these nanocomposite particles collapsed faster than the other particles. In the particles prepared using 2.00 and $4.00 \%(\mathrm{w} / \mathrm{v})$ PVA aqueous solution, 40 min after the tests' initiation, the cumulative release ratios of RFX reached $85.0 \pm 9.5 \%$ and $82.8 \pm 5.8 \%$, respectively. It is challenging to obtain molecularly dispersed PVA solutions in water ${ }^{34)}$ PVA, which could not be adsorbed on the particle surface, physically mixed with the amino acids, and prevented their dissolution. The cumulative release ratio of RFX from the particles prepared using purified water did not reach 50\% even after $1 \mathrm{~h}$ from the tests' initiation. We visually confirmed the presence of unbroken nanocomposite particles.

\subsection{In vitro release of RFX from redispersed RFX-loaded PLLGA nanoparticles}

The cumulative release ratios of RFX-loaded PLLGA nanoparticles prepared using purified water, $0.25,0.50$, 2.00 , or $4.00 \%(\mathrm{w} / \mathrm{v}) \mathrm{PVA}$ aqueous solution at $37^{\circ} \mathrm{C}$ are shown in Fig. 5. For all types of particles, the release ratios reached $97 \% 8 \mathrm{~h}$ after the initiation of the tests (Fig. 5a). Focusing on the early stages of drug release, we confirmed that after 0.5 and $1 \mathrm{~h}$ from the initiation of the tests, the release ratios of the nanoparticles prepared using 0.50 and $2.00 \%(\mathrm{w} / \mathrm{v}) \mathrm{PVA}$ aqueous solution were significantly lower than those of the others (Fig. 5b). RFX is a hydrophobic drug and is almost insoluble in water ${ }^{35)}$. These results indicate that the PVA-derived hydrated layer on their surfaces suppressed RFX release from the nanoparticles. However, the nanoparticles prepared using a $4.00 \%(\mathrm{w} / \mathrm{v}) \mathrm{PVA}$ aqueous solution showed high release ratios similar to those prepared using purified water and a $0.25 \%(\mathrm{w} / \mathrm{v})$ PVA aqueous solution. PVA has been reported to effectively increase the apparent solubility of poorly water-soluble drugs by functioning as a concentration-enhancing polymer ${ }^{24)}$. Therefore, we considered that the presence of a large amount of PVA in the poor solvent increased the apparent solubility of RFX in water, and some RFX was transferred to water without being contained in the particles. The RFX transferred into the water was contained in the nanocomposite particles' amino acid portion and caused high drug release ratios in the early stages of drug release. 


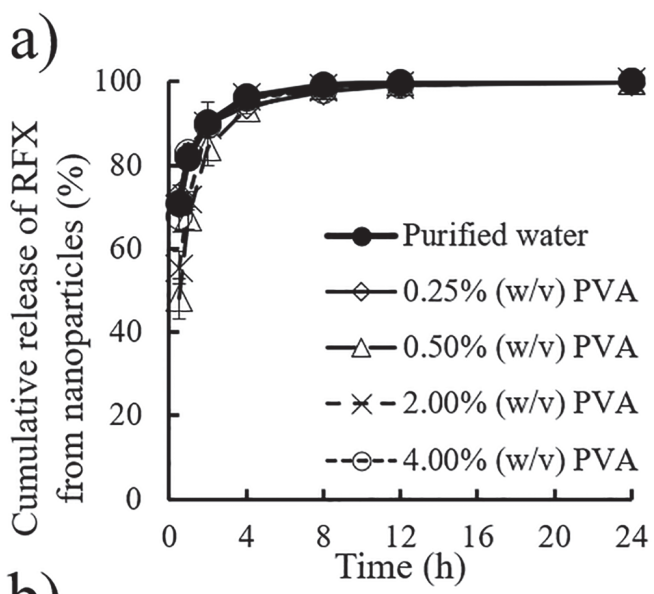

b)

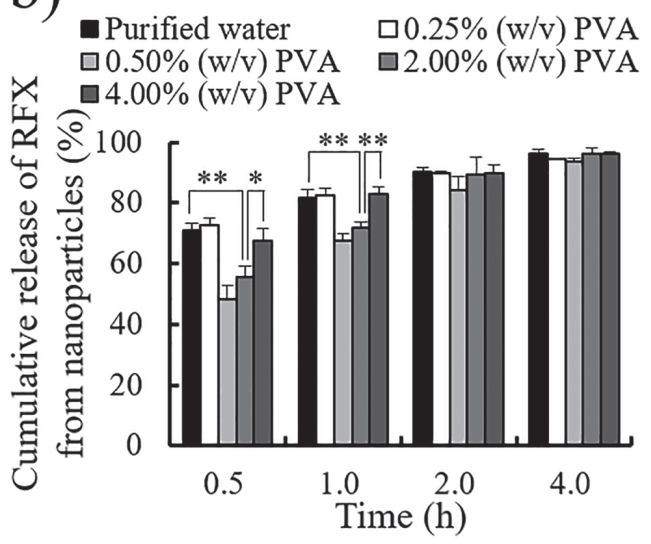

Fig. 5 Cumulative release ratios of RFX from PLLGA nanoparticles prepared using purified water, 0.25, $0.50,2.00$, and $4.00 \%(\mathrm{w} / \mathrm{v})$ PVA aqueous solution after $0.5-24 \mathrm{~h}$ from the initiation of the tests (a) and 0.5-4 $\mathrm{h}$ from the initiation of the tests (b) (mean \pm S.D., $\mathrm{n}=3,{ }^{*} p<0.05,{ }^{*} p<0.01$, Tukey's test).

\section{Conclusions}

In this study, we controlled the drug release behavior of nanocomposite particles using PVA. The nanocomposite particles prepared using a $0.50 \%(\mathrm{w} / \mathrm{v})$ PVA aqueous solution succeeded in the rapid release of nanoparticles from nanocomposite particles and suppressing the initial burst. These particles can be expected to redisperse into nanoparticles after reaching the lungs rapidly. This property is similar to that of nanocomposite particles prepared using conventional sugars. Therefore, these particles could be used to treat lung diseases and the stable storage of nanoparticles. Because the lungs have less water than the release test conditions used in this study, it may be necessary to develop nanocomposite particles that more easily release nanoparticles. Nanocomposite particles prepared using $0.25-2.00 \%(w / v)$ PVA aqueous solution should be studied in detail. The nanocomposite particles prepared using a $2.00 \%(\mathrm{w} / \mathrm{v})$ PVA aqueous solution succeeded in the sustained release of nanoparticles from nanocomposite particles and suppressing the initial burst. In addition to the release of the drug from the nanoparticles, these particles can be expected to be sustained-release in releasing the nanoparticles from the nanocomposite particles. The particles may reduce the frequency of drug administration in diseases that require long-term treatment, such as tuberculosis and lung cancer. This study conducted release tests on nanoparticles redispersed from nanocomposite particles to investigate the particles and drug behavior after reaching the lungs. In the future, to examine the effect of PVA on drug release behavior from nanoparticles in detail, it may be necessary to focus on nanoparticles immediately after preparation and nanoparticles from which surrounding drugs have been removed after redispersion.

\section{Acknowledgment}

The authors are grateful for the suggestions given by Mr. T. Nakajima from Tokyo University of Science.

\section{Conflict of Interest}

The authors declare that they have no potential conflicts of interest.

\section{References}

1) Takeuchi, I.; Kimura, Y.; Makino, K. Effect of conformation of poly (L-lactide-co-glycolide) molecules in organic solvents on nanoparticle size. J. Oleo Sci. 69, 1125-1132(2020).

2) Prausnitz, M.R.; Langer, R. Transdermal drug delivery. Nature Biotech. 26, 1261-1268(2008).

3) Köhler, D. Aerosols for systemic treatment. Lung 168, 677-684(1990).

4) Taki, M.; Tagami, T.; Fukukshige, K.; Ozeki, T. Fabrication of nanocomposite particles using a two-solution mixing-type spray nozzle for use in an inhaled curcumin formulation. Int. J. Pharm. 511, 104-110 (2016).

5) Edwards, D.A.; Ben-Jebria, A.; Langer, R. Recent advances in pulmonary drug delivery using large, porous inhaled particles. J. Appl. Physiol. 85, 379-385 (1998).

6) Schürch, S.; Gehr, P.; Im Hof, V.; Geiser, M.; Green, F. Surfactant displaces particles toward the epithelium in airways and alveoli. Resp. Physiol. 80, 17-32 (1990).

7) Ghirardelli, R.; Bonasoro, R.; Porta, C.; Cremaschi, D. Identification of particular epithelial areas and cells that transport polypeptide-coated nanoparticles in the nasal respiratory mucosa of the rabbit. Biochim. Bio- 


\section{Effects of Polyvinyl Alcohol on Nanocomposite Particles}

phys. Acta 1416, 39-47(1999).

8) Russell-Jones, G.J.; Veitch, H.; Arthur, L. Lectin-mediated transport of nanoparticles across Caco-2 and OK cells. Int. J. Pharm. 190, 165-174(1999).

9) Huang, M.; Ma, Z.; Khor, E.; Lim, L.-Y. Uptake of FITCchitosan nanoparticles by A549 cells. Pharm. Res. 19, 1488-1494 (2002).

10) Tomoda, K.; Ohkoshi, T.; Kawai, Y.; Nishiwaki, M.; Nakajima, T.; Makino, K. Preparation and properties of inhalable nanocomposite particles: Effects of the temperature at a spray-dryer inlet upon the properties of particles. Colloids Surf. B 61, 138-144(2008).

11) Ghasemian, E.; Vatanara, A.; Rouini, M.R.; Najafabadi, A.R.; Gilani, K.; Lavasani, H.; Mohajel, N. Inhaled sildenafil nanocomposites: Lung accumulation and pulmonary pharmacokinetics. Pharm. Dev. Tech. 21, 961971 (2016).

12) Takeuchi, I.; Koshi, Y.; Makino, K. Drug delivery properties of nanocomposite particles for inhalation: Comparison of drug concentrations in lungs and blood. In Vivo 34, 543-547(2020).

13) Menon, J.U.; Ravikumar, P.; Pise, A.; Gyawali, D.; Hsia, C.C.; Nguyen, K.T. Polymeric nanoparticles for pulmonary protein and DNA delivery. Acta Biomater. 10, 2643-2652 (2014).

14) Takeuchi, I.; Tomoda, K.; Hamano, A.; Makino, K. Effects of physicochemical properties of poly (lactide-coglycolide) on drug release behavior of hydrophobic drug-loaded nanoparticles. Colloids. Surf. A 520, 771778 (2017).

15) Takeuchi, I.; Yamaguchi, S.; Goto, S.; Makino, K. Drug release behavior of hydrophobic drug-loaded poly (lactide-co-glycolide) nanoparticles: Effects of glass transition temperature. Colloids. Surf. A 529, 328-333 (2017).

16) Rad, R.T.; Dadashzadeh, S.; Vatanara, A.; Alavi, S.; Ghasemian, E.; Mortazavi, S.A. Tadalafil nanocomposites as a dry powder formulation for inhalation, a new strategy for pulmonary arterial hypertension treatment. Eur. J. Pharm. Sci. 133, 275-286(2019).

17) Nishino, M.; Tagami, T.; Taki, M.; Ozeki, T. Effective spray drying technique to prepare nanocomposite particles by preventing the growth of needle-like simvastatin crystal. J. Drug Deliv. Sci. Tech. 53, 101188 (2019).

18) Malamatari, M.; Somavarapu, S.; Bloxham, M.; Buckton, G. Nanoparticle agglomerates of indomethacin: The role of poloxamers and matrix former on their dissolution and aerosolisation efficiency. Int. J. Pharm. 495, 516-526 (2015).

19) Takeuchi, I.; Tetsuka, Y.; Nii, T.; Shinogase, M.; Makino, $\mathrm{K}$. Inhalable nanocomposite particles using amino acids with improved drug content and humidity resistance. Colloids. Surf. A 529, 387-393(2017).
20) Mehanna, M.M.; Mohyeldin, S.; Elgindy, N.A. Rifampicin-carbohydrate spray-dried nanocomposite: a futuristic multiparticulate platform for pulmonary delivery. Int. J. Nanomedicine 14, 9089-9112(2019).

21) Galindo-Rodriguez, S.; Allemann, E.; Fessi, H.; Doelker, E. Physicochemical parameters associated with nanoparticle formation in the salting-out, emulsification-diffusion, and nanoprecipitation methods. Pharm. Res. 21, 1428-1439(2004).

22) McDonald, C.; Kaye, S.; Figueiredo, F.; Macintosh, G.; Lockett, C. A randomised, crossover, multicentre study to compare the performance of $0.1 \%(\mathrm{w} / \mathrm{v})$ sodium hyaluronate with $1.4 \%(\mathrm{w} / \mathrm{v})$ polyvinyl alcohol in the alleviation of symptoms associated with dry eye syndrome. Eye 16, 601-607 (2002).

23) Riis, T.; Bauer-Brandl, A.; Wagner, T.; Kranz, H. pH-independent drug release of an extremely poorly soluble weakly acidic drug from multiparticulate extended release formulations. Eur. J. Pharm. Biopharm. 65, 78-84 (2007).

24) Brough, C.; Miller, D.A.; Keen, J.M.; Kucera, S.A.; Lubda, D.; Eilliams III, R.O. Use of polyvinyl alcohol as a solubility-enhancing polymer for poorly water soluble drug delivery (part I). AAPS Pharm. Sci. Tech. 17, 167-179 (2016).

25) Tomoda, K.; Yabuki, N.; Terada, H.; Makino, K. Surfactant free preparation of PLGA nanoparticles: the combination of antisolvent diffusion with preferential solvation. Colloids Surf. A 457, 88-93 (2014).

26) Kogawa, A.C.; Salgado, H.R.N. Rifaximin stability: A look at UV, IR, HPLC, and turbidimetry method. J. AOAC Int. 101, 410-413 (2018).

27) Fessi, H.; Puisieux, F.; Devissaguet, J.Ph.; Ammoury, N.; Benita, S. Nanocapsule formation by interfacial polymer deposition following solvent displacement. Int. J. Pharm. 55, R1-R4(1989).

28) Takeuchi, I.; Kanno, Y.; Makino, K. Polyborane-encapsulated PEGylated liposomes prepared using post-insertion technique for boron neutron capture therapy. J. Oleo Sci. 68, 1261-1270(2019).

29) Takeuchi, I.; Kagawa, A.; Makino, K. Skin permeability and transdermal delivery route of 30-nm cyclosporin A-loaded nanoparticles using PLGA-PEG-PLGA triblock copolymer. Colloids Surf. A 600, 124866 (2020).

30) Takeuchi, I.; Suzuki, T.; Makino, K. Skin permeability and transdermal delivery route of 50-nmindomethacin-loaded PLGA nanoparticles. Colloids Surf. B 159, 312-317 (2017).

31) Tomoda, K.; Ohkoshi, T.; Nakajima, T.; Makino, K. Preparation and properties of inhalable nanocomposite particles: Effects of the size, weight ratio of the primary nanoparticles in nanocomposite particles and temperature at a spray-dryer inlet upon properties of nanocomposite particles. Colloids Surf. B 64, 70-76 
(2008).

32) Takeuchi, I.; Taniguchi, Y.; Tamura, Y.; Ochiai, K.; Makino, K. Effects of L-leucine on PLGA microparticles for pulmonary administration prepared using spray drying: Fine particle fraction and phagocytotic ratio of alveolar macrophages. Colloids Surf. A 537, 411-417 (2018).

33) Sahoo, S.K.; Panyam, J.; Prabha, S.; Labhasetwar, V. Residual polyvinyl alcohol associated with poly (D,Llactide-co-glycolide) nanoparticles affects their physical properties and cellular uptake. J. Control. Release
82, 105-114(2002).

34) Tacx, J.C.J.F.; Schoffeleers, H.M.; Brands, A.G.M.; Teuwen, L. Dissolution behavior and solution properties of polyvinylalcohol as determined by viscometry and light scattering in DMSO, ethyleneglycol and water. Polymer 41, 947-957 (2000).

35) Laterza, L.; Ianiro, G.; Scoleri, I.; Landi, R.; Bruno, G.; Scaldaferri, F.; Gaetani, E.; Campanale, M.; Gasbarrini, A. Rifaximin for the treatment of diarrhoea-predominant irritable bowel syndrome. Expert Opin. Pharmacother. 16, 607-615(2015). 